\title{
Premature ventricular complexes as a first manifestation in left ventricular non-compaction cardiomyopathy: A case report and focused review of the literature
}

\author{
Cristian Orlando Porras Bueno ${ }^{1}$, Alejandro Sánchez Velásquez ${ }^{1}$, and Alexander Álvarez \\ Ortiz $^{2}$ \\ ${ }^{1}$ Universidad Autónoma de Bucaramanga \\ ${ }^{2}$ Instituto del Corazón de Bucaramanga
}

February 2, 2022

\begin{abstract}
Left ventricular non-compaction (LVNC) cardiomyopathy is an uncommon unclassified or genetic myocardial disorder. Frequent premature ventricular complexes (PVCs) as unique finding in LVNC cardiomyopathy are rare. We report a case of a 36-year-old woman in whom isolated LVNC was diagnosed due to an incidental finding of PVCs in pre-operative consultation.
\end{abstract}

\section{Introduction:}

Isolated LVNC is characterized by prominent LV trabeculae and deep intertrabecular recesses which are filled with blood from the ventricular cavity without evidence of communication to the epicardial coronary artery system. Additionally, a thin compacted layer of myocardium is present. It has been cataloged as an unclassified cardiomyopathy by the European Society of Cardiology (ESC), while the American Heart Association (AHA) classifies it as a genetic cardiomyopathy ${ }^{1-2}$. About its pathogenesis, a congenital and an acquired basis have been proposed. The former, states that during the embryological heart development process the myocardial compaction is disrupted through unknow mechanisms; and the latter that LVNC could be acquired and developed on time ${ }^{3}$. Besides, trabeculations that could fulfill criteria for LVNC have been described in athletes, pregnancy and patients with chronically increased LV pre-load and afterload diseases like heart valve disease, chronic renal failure and sickle cell disease ${ }^{3}$.

Nevertheless, a family history has been described in a significant proportion of patients with an autosomal dominant X-linked transmission ${ }^{4}$. Furthermore, several gene mutations have been reported primarily in genes coding for sarcomeric, cytoskeletal, Z-line and mitochondrial proteins ${ }^{4}$. The first mutation described was in the tafazzin gene, although others like MYH7, RYR2 and lamin A/C have been reported. These findings suggest that also genetics is involved in LVNC development ${ }^{4}$. Consequently, current research evidence points towards to its reclassification as a distinct phenotype resulting from the interaction between the environment and genetics ${ }^{5}$.

LVNC prevalence in general population is not known, but it has been described between 0.014 and $1.3 \%$ in patients undergoing echocardiography ${ }^{6-8}$. Even though it has been reported in some cases, the right ventricular non compaction (RVNC) real prevalence is unknow maybe due to its unclear definition and recognition ${ }^{3}$. Nowadays, there is no gold standard for LVNC diagnosis, however echocardiography and cardiac magnetic resonance (CMR) are the best diagnostic tests currently available ${ }^{3}$.

Clinical manifestations of the LVNC cardiomyopathy are multiple and non-specific, including dyspnea, chest pain, palpitations and syncope. Furthermore, non-sustained ventricular tachycardia (VT), left bundle branch 
block (LBBB) and atrial fibrillation (AF) have been described as the most frequent electrocardiographic findings ${ }^{8-11}$. Herein, we describe a case of frequent PVCs as first manifestation of a patient with LVNC cardiomyopathy.

\section{Case report}

A 36-year-old woman in whom a left anterior cruciate ligament repairment was planned, reported a history of 3 months of palpitations and dyspnea in the pre-anesthetic examination, where arrhythmic heart sounds were found, reason why she was referred for cardiologist evaluation.

An initial electrocardiogram (ECG) showed sinus rhythm without any abnormality and a normal echocardiogram was obtained. The 24 hours Holter-ECG showed frequent PVCs with an arrhythmic burden of $35 \%$ (Figure 1), so $\beta$-blocker therapy was prescribed. Ischemic substrate was ruled-out with a dobutamine stress-echocardiogram as well as Chagas disease and electrolyte disturbances. However, the CMR revealed prominent trabeculae and deep recesses in the left ventricle (LV) apex, as well as in the anterior, inferior and lateral wall of the LV. The right ventricle (RV) was normal without any myocardial or function disturbances (Figure 2). Therefore, the diagnosis of isolated LVNC cardiomyopathy was proposed.

Despite the prescription of highest-tolerated dose of $\beta$-blocker therapy, it was recorded a persistent high PVCs burden $(22 \%)$ in a 24 hours Holter-ECG; reason why the patient was referred to the Electrophysiologist for ventricular ablation. On the procedure day, the baseline ECG of the patient showed a sinus rhythm with right ventricular outflow tract (RVOT) complexes in ventricular bigeminy. The preliminary electrophysiology study found normal sinus function test, normal atrioventricular conduction intervals and absence of accessory pathways. Then a Pentarray Biosense@ catheter was used to obtain a RVOT-3D electroanatomic mapping with the CARTO-3 mapping system.

The activation map identified the PVCs origin in the anterolateral segment of the RVOT (Figure 3) where the topostimulation with pass mode and pattern-matching showed a correlation of the ninety five percent compared with the extrasystole. Then, radiofrequency ablation was performed. Afterwards, no tachycardia was induced with the programmed auricular and ventricular heart stimulation. No complications related to the procedure were reported and the patient was discharged from the hospital on the next day, with her previous antiarrhythmic medication. In the follow-up after 3 months of the ventricular ablation, remains asymptomatic and free of PVCs.

\section{Discussion}

The diagnosis of LVNC is usually stablished by identifying the morphologic diagnostic criteria proposed by Jenni et al. on transthoracic echocardiography, which are the most validated and commonly used ${ }^{12}$. Those include: 1) Absence of coexisting cardiac abnormalities (by definition); 2) A two-layer structure with a compacted thin epicardial band and a much thicker non-compacted endocardial layer of trabecular meshwork with deep endomyocardial spaces. A maximal end systolic ratio of non-compacted to compacted layers of $>2$; 3) A predominant localization of the affected segments at the mid-lateral, mid-inferior and apical areas. Concomitant regional hypokinesia was not confined to the non-compacted segments; 4) Colour Doppler evidence of deep perfused intertrabecular recesses; 5) a Compacted wall thickness under or equal to $8.1 \mathrm{~mm}^{13-15}$.

Despite this, sometimes the echocardiographic findings are not diagnostic or are inconclusive as occurred in our patient, and a CMR should be performed to confirm the diagnosis. Anyway, the CMR criteria for diagnosis of LVNC differ slightly from the echocardiographic criteria, since an end-diastolic noncompacted to compacted ratio $>2.3$ measured in the CMR at basal, mid and apical segments has been considered the cutoff and the most accurate morphologic criteria for LVNC diagnosis ${ }^{3,16-18}$. The CMR criteria was fulfilled in our case with an end-diastolic non compacted to compacted ratio $>3$.

Although LVNC could be associated with other cardiomyopathies and congenital cardiac defects ${ }^{19-24}$, it also could be found in highly trained athletes, patients with sickle cell anemia and pregnants ${ }^{25-28}$. However, as we described in our patient, LVNC could be seen in the absence of these anomalies and is referred as isolated LVNC. 
LVNC cardiomyopathy complications include ventricular and atrial arrhythmias, sudden death, systemic embolism and also heart failure which is the most frequently complication described among several series in children and adults ${ }^{8-11}$. Abnormal electrocardiographic findings related but not specific to LVNC are left or right bundle brunch blocks, fascicular blocks, repolarization abnormalities such as T-Wave inversion and ST-Segment changes high-degree atrioventricular block, AF, atrial flutter, VT and Wolff-ParkinsonWhite syndrome mainly in children ${ }^{8-11}$. Frequent PVCs symptoms-related as a first manifestation of LVNC are very unusual ${ }^{8-11}$. Potentially, non-compaction itself may represent a proarrhythmogenic substrate and therefore, several mechanisms have been proposed to explain this including: concurrent development of arrest of the conduction system, intertrabecular crypts creating pathways for reentrant circuits, and ischemia from epicardial coronary hypoperfusion of trabeculations ${ }^{28}$.

Up to date, there is no specific guidelines for management of LVNC. Its diagnosis requires to rule-out differential diagnoses as prominent hypertrabeculation with normal compacted LV layer, hypertrophic or dilated cardiomyopathy, endocardial fibroelastosis and LV apical thrombus among others. Its management should include clinical monitoring for asymptomatic patients with normal LV size and function or clinical guidance according to current therapeutic evidence in symptomatic patients due to LV dysfunction and/or arrythmias. Genetic testing for LVNC is not always available and it should not change the clinical management of the disease, but it may be useful for LVNC diagnosis screening in patient's relatives ${ }^{3}$.

\section{Conclusion}

PVCs could be the first manifestation of LVNC cardiomyopathy. Thus, physicians must always pay attention to this "benign" arrythmia, especially when a high arrhythmic burden is detected, in order to allow a proper diagnosis of a potential cardiomyopathy and to establish accurately its management and treatment.

\section{Acknowledgments:}

The authors thank all individuals who participated in this study. Published with written consent of the patient.

\section{Conflict of interest:}

The authors report no conflict of interest.

\section{Author contributions}

Alejandro Sanchez Velasquez: Studied the conception and designed the study, also reviewed the manuscript. Alexander Alvarez Ortiz: Studied the conception and designed the study, also reviewed the manuscript.

Cristian Orlando Porras Bueno: Wrote, reviewed the manuscript and also was involved in acquisition of data and analysis of data.

\section{References}

1. Maron BJ, Towbin JA, Thiene G, Antzelevitch C, Corrado D, Arnett D, et al. Contemporary definitions and classification of the cardiomyopathies: An American Heart Association Scientific Statement from the Council on Clinical Cardiology, Heart Failure and Transplantation Committee; Quality of Care and Outcomes Research and Functional Genomics and Translational Biology Interdisciplinary Working Groups; and Council on Epidemiology and Prevention. Circulation. 2006;113(14):1807-16.

2. Elliott P, Andersson B, Arbustini E, Bilinska Z, Cecchi F, Charron P, et al. Classification of the cardiomyopathies: A position statement from the european society of cardiology working group on myocardial and pericardial diseases. Eur Heart J. 2008;29(2):270-6.

3. Arbustini E, Weidemann F, Hall JL. Left ventricular noncompaction: A distinct cardiomyopathy or a trait shared by different cardiac diseases? J Am Coll Cardiol. 2014;64(17):1840-50. 
4. Pascale R, Flavie A, Roux M, Donal E, Eicher J-C, Aoutil N, et al. Targeted panel sequencing in adult patients with left ventricular non-compaction reveals a large genetic heterogeneity. Clin Genet. 2019;95(3):35667.

5. Hershberger RE, Morales A, Cowan J. Is Left Ventricular Noncompaction a Trait, Phenotype, or Disease? The Evidence Points to Phenotype. Circ Cardiovasc Genet. 2017;10(6):1-3.

6. Aras D, Tufekcioglu O, Ergun K, Ozeke O, Yildiz ALI, Topaloglu S, et al. Clinical Features of Isolated Ventricular Noncompaction in Adults Long-Term Clinical Course, Echocardiographic Properties , and Predictors of Left Ventricular Failure. J Card Fail. 2006;12(9):726-33.

7. Stanton C, Bruce C, Connolly H, Brady P, Syed I, Hodge D, et al. Isolated Left Ventricular Noncompaction Syndrome. Am J Cardiol [Internet]. 2009;104(8):1135-8. Available from:http://dx.doi.org/10.1016/j.amjcard.2009.05.062.

8. Oechslin EN, Jost CHA, Rojas JR, Kaufmann PA, Jenni R. Long-Term Follow-up of 34 Adults With Isolated Left Ventricular Noncompaction : A Distinct Cardiomyopathy With Poor Prognosis. J Am Coll Cardiol. 2000;36(2):493-500.

9. Pignatelli RH, Mcmahon CJ, Dreyer WJ, Denfield SW, Price J, Belmont JW, et al. Clinical Characterization of Left Ventricular Noncompaction in Children A Relatively Common Form of Cardiomyopathy. Circulation. 2003;108:2672-9.

10. Brescia ST, Rossano JW, Pignatelli R, Jefferies JL, Price JF, Decker JA, et al. Mortality and Sudden Death in Pediatric Left Ventricular Noncompaction in a Tertiary Referral Center. Circulation. $2013 ; 127: 2202-8$.

11. Bhatia NL, Tajik AJ, Wilansky S, Steidley DE. Isolated Noncompaction of the Left Ventricular Myocardium in Adults : A Systematic Overview. J Card Fail [Internet]. 2011;17(9):771-8. Available from:http://dx.doi.org/10.1016/j.cardfail.2011.05.002

12. Gati S, Rajani R, Carr-White GS, Chambers JB. Adult left ventricular noncompaction: Reappraisal of current diagnostic imaging modalities. JACC Cardiovasc Imaging. 2014;7(12):1266-75.

13. Jenni R, Oechslin E, Schneider J, Jost CA, Kaufmann PA. Echocardiographic and pathoanatomical characteristics of isolated left ventricular non-compaction : a step towards classification as a distinct cardiomyopathy. Heart. 2001;86:666-71

14. Frischknecht BS, Jost CHA, Oechslin EN, Seifert B, Hoigne P, Roos M, et al. Validation of Noncompaction Criteria in Dilated Cardiomyopathy, and Valvular and Hypertensive Heart Disease. J Am Soc Echocardiogr. 2005;18:865-72.

15. Cardiomyopathy N, Gebhard C, St BE, Greutmann M, Biaggi P, Jenni R, et al. Reduced Left Ventricular Compacta Thickness : A Novel Echocardiographic Criterion for Non-Compaction Cardiomyopathy. J Am Soc Echocardiogr. 2012;25:1050-7.

16. Petersen SE, Selvanayagam JB, Wiesmann F, Robson MD, Francis JM, Anderson RH, et al. Left Ventricular Non-Compaction Insights From Cardiovascular Magnetic Resonance Imaging. J Am Coll Cardiol. 2005;46(1):101-5.

17. Fazio G, Novo G, D'angelo L, Visconti C, Sutera L, Grassedonio E, et al. Magnetic resonance in isolated noncompaction of the ventricular myocardium. Int J Cardiol [Internet]. 2008;140(3):367-9. Available from:http://dx.doi.org/10.1016/j.ijcard.2008.11.080

18. Stollberger C, Kopsa W, Tscherney R, Finsterer J. Diagnosing Left Ventricular Noncompaction by Echocardiography and Cardiac Magnetic Resonance Imaging and Its Dependency on Neuromuscular Disorders. Clin Cardiol. 2008;31(8):383-7. 
19. Biagini E, Ragni L, Ferlito M, Pasquale F, Lofiego C, Leone O, et al. Different Types of Cardiomyopathy Associated With Isolated Ventricular Noncompaction. Am J Cardiol. 2006;98:821-4.

20. Wlodarska EK, Wozniak O, Konka M, Piotrowska-kownacka D, Walczak E, Hoffman P. Isolated ventricular noncompaction mimicking arrhythmogenic right ventricular cardiomyopathy - A study of nine patients. Int J Cardiol [Internet]. 2009;145(1):107-11. Available from:http://dx.doi.org/10.1016/j.ijcard.2009.05.062

21. Azevedo O, Gaspar P, Sa Miranda C, Cunha D, Medeiros R, Lourenco A. Left Ventricular Noncompaction in a Patient with Fabry Disease : Overdiagnosis, Morphological Manifestation of Fabry Disease or Two Unrelated Rare Conditions in the Same Patient ? Cardiology. 2011;119:155-9.

22. Oechslin E, Jenni R. Left ventricular non-compaction revisited : a distinct phenotype with genetic heterogeneity ? Eur Heart J. 2011;32:1446-56.

23. Paterick TE, Tercius AJ, Agarwak A, Treiber SC, Khandheria BK, Tajik AJ. Double Jeopardy in the Echocardiography Laboratory : Coexistence of Two Distinct Cardiomyopathies ? Echocardiography. 2014;31(8):931-5.

24. Rapezzi C, Leone O, Ferlito M, Biagini E, Coccolo F, Arpesella G. Isolated ventricular non-compaction with restrictive cardiomyopathy. Eur Heart J. 2006;27(16):1927.

25. Gati S, Chandra N, Bennett RL, Reed M, Kervio G, Panoulas VF, et al. Increased left ventricular trabeculation in highly trained athletes : do we need more stringent criteria for the diagnosis of left ventricular non-compaction in athletes? Heart. 2013;99:401-8.

26. Gati S, Papadakis M, Papamichael ND, Zaidi A, Sheikh N, Reed M, et al. Reversible De Novo Left Ventricular Trabeculations in Pregnant Women : Implications for the Diagnosis of Left Ventricular NonCompaction in Low Risk Populations. Circulation. 2014;130(6):475-83.

27. Kohli SK, Pantazis AA, Shah JS, Adeyemi B, Jackson G, Mckenna WJ, et al. Diagnosis of leftventricular non-compaction in patients with left-ventricular systolic dysfunction : time for a reappraisal of diagnostic criteria ? Eur Heart J. 2008;29:89-95.

28. Gati S, Papadakis M, Niekerk N Van, Reed M, Yeghen T, Sharma S. Increased left ventricular trabeculation in individuals with sickle cell anaemia : Physiology or pathology ? Int J Cardiol [Internet]. 2013;168(2):1658-60.

\section{Figures}

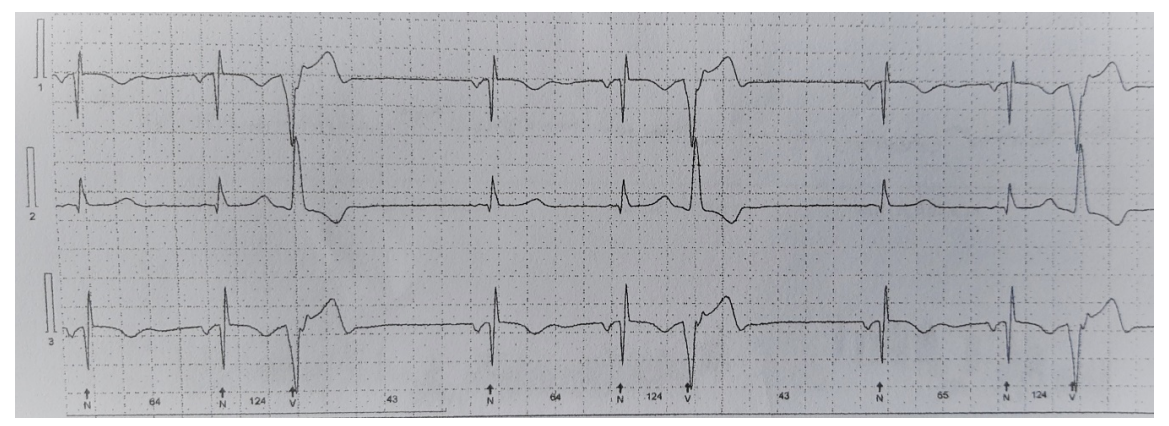

Figure 1. Holter monitor showing PVCs in trigeminy pattern. PVCs $=$ Premature ventricular complexes. 


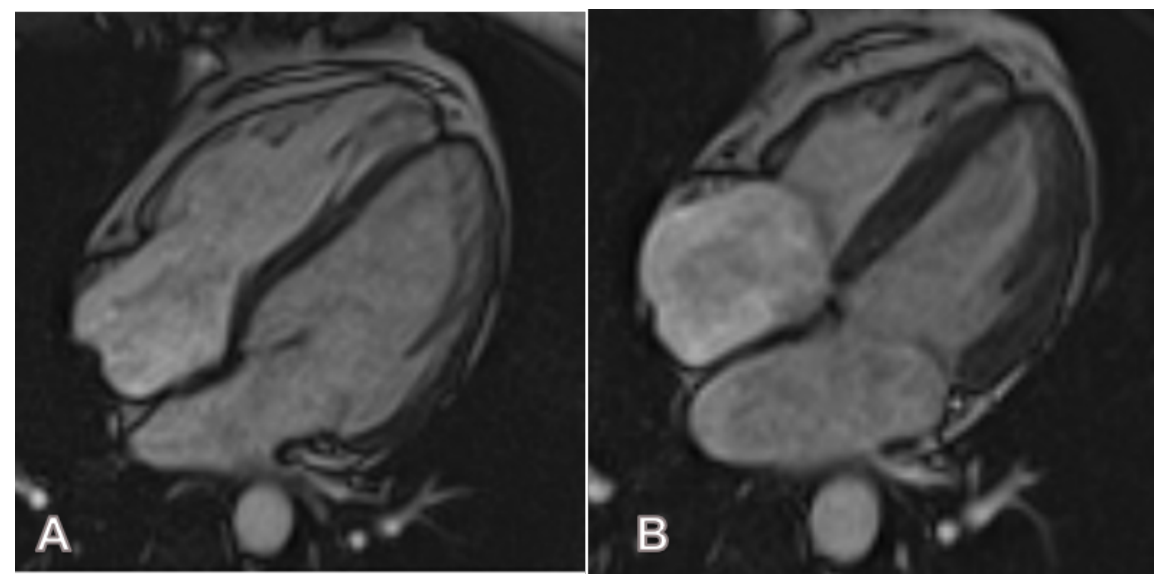

Figure 2. A. CMR cine sequence showing prominent trabeculae and deep recesses (yellow arrows) in the LV apex and lateral wall and a maximum end-diastolic noncompacted to compacted myocardial thickness ratio of $>2.3$ B. LV noncompacted to compacted myocardial thickness ratio $>2$ at the end of the systole. $\mathrm{CMR}=$ Cardiac magnetic resonance. $\mathrm{LV}=$ Left ventricular.

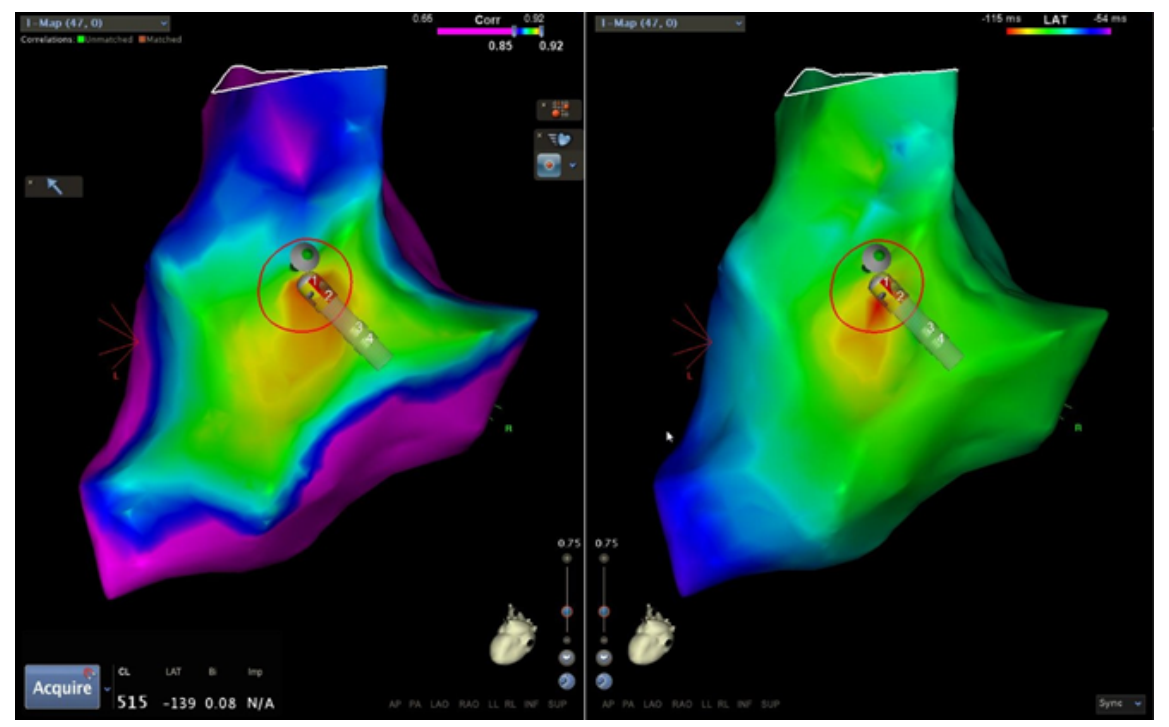

Figure 3. Voltage map with 3D CARTO-3 system showing primo activation zone in the anterolateral segment of the right ventricular tract outflow, where radiofrequency ablation was performed. 


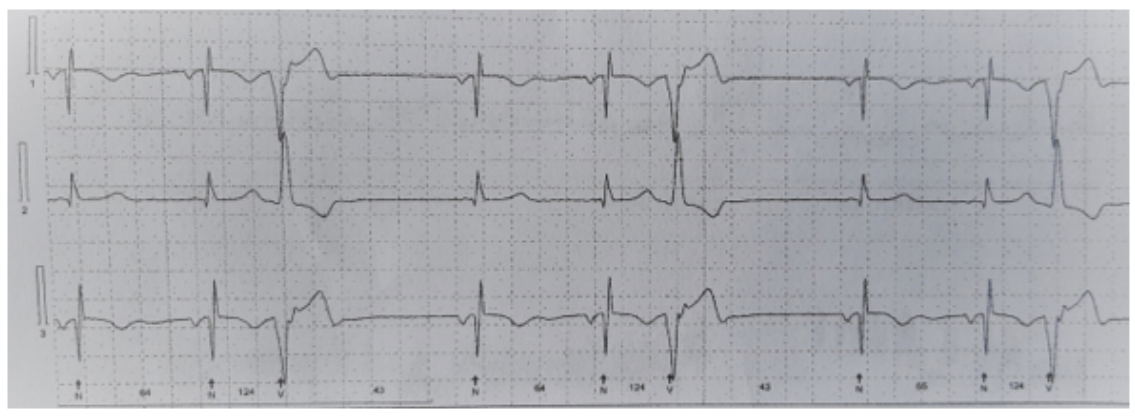

Figure 1. Holter monitor showing PVCs in trigeminy pattern. PVCs = Premature ventricular complexes.
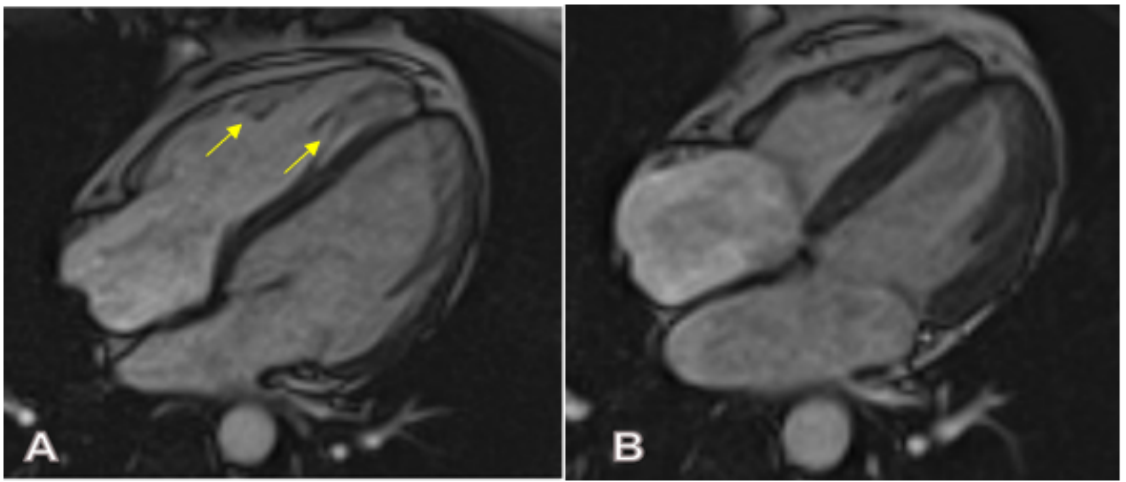

Figure 2. A. CMR cine sequence showing prominent trabeculae and deep recesses (yellow arrows) in the LV apex and lateral wall and a maximum end-diastolic noncompacted to compacted myocardial thickness ratio of $>2.3 \mathrm{~B}$. LV noncompacted to compacted myocardial thickness ratio $>2$ at the end of the systole. $C M R=$ Cardiac magnetic resonance. $L V=$ Left ventricular. 


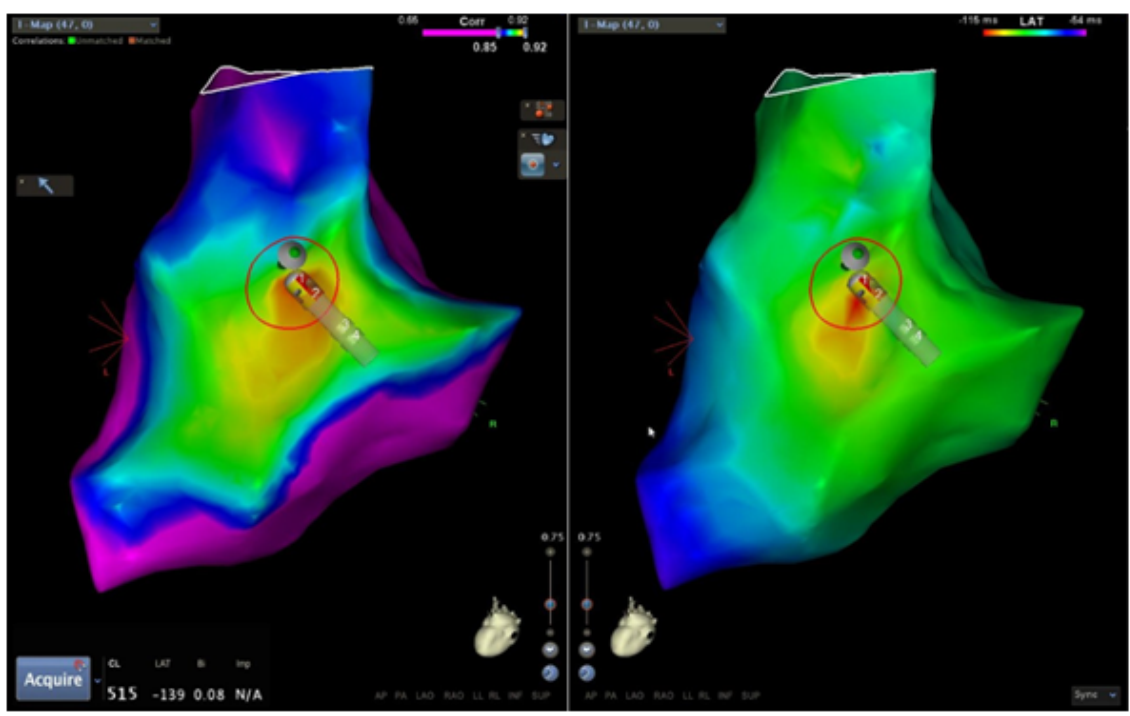

Figure 3. Voltage map with 3D CARTO-3 system showing primo activation zone in the anterolateral segment of the right ventricular tract outflow, where radiofrequency ablation was performed. 